ISSN (e)-2347-176x ISSN (p) 2455-0450

crossrefDOI: https://dx.doi.org/10.18535/jmscr/v7i1.101

\title{
Absolute CD34+ Cell Count by Flow Cytometry at M.Y. Hospital Indore
}

Authors

\author{
Prof. Dr C.V. Kulkarni ${ }^{1}$, Prof. Dr A. Panchonia ${ }^{2}$, Dr Sachin Sharma ${ }^{3}$ \\ ${ }^{1}$ Head of Pathology Department, MGM Medical College with M.Y. Hospital Indore MP \\ ${ }^{2}$ Professor, MGM Medical College with M.Y. Hospital Indore MP \\ ${ }^{3}$ Assistant Professor MGM Medical College with M.Y. Hospital Indore, MP \\ Corresponding Author \\ Dr Sachin Sharma
}

Assistant Professor MGM Medical College with M.Y. Hospital Indore, MP, India

Email: sachinchanderi@gmail.com

\begin{abstract}
Introduction: An increasing number of medical centers can collect bone marrow, peripheral blood, stem cells. Pathology laboratories should accommodate this trend, but investment in additional equipment may be impractical. In concert with the International Society of Hematotherapy and Graft Engineering (ISHAGE), we previously described a set of guidelines for detection of CD34cells With this procedure, an absolute CD34 count is generated by incorporating the leukocyte count from an automated hematology analyser (twoplatform method). In the present study, we modified the basic ISHAGE method with the addition of a known number of Flow-County fluorospheres. To reduce errors inherent to sample washing/centrifugation,

Aims: To compare CD34+ cell counting results by using with and without the use of a separate hematology analyzer (ie, single-platform versus dual-platform methodologies).

Material and Method: A total of 34 samples of peripheral blood, apheresis packs, and bone marrow aspiration were analysed and compared using the ISHAGE protocol with or without the addition of fluorescent microspheres. Dual-platform CD34+ cell counting incorporated data from navious modal 2 laser and eight colour.

Results: Subtle differences in CD34+ cell counting between 2 systems and 2 methods did not achieve statistical significance.

Conclusion: It is the accurate determination CD34+ cell enumeration, properly validated, can support care for patients undergoing transplants setting. These modifications may improve the inter laboratory reproducibility

Keywords: International Society of Hematotherapy and Graft Engineering (ISHAGE), Absolute CD34+ Cell COUNT.
\end{abstract}

\section{Introduction}

The success of bone marrow or peripheral blood stemcell (PBSC) and bone marrow transplants is associated not only with phenotypic compatibility between donor and patient but also with the number of CD34+ white cells in the graft, relative to body weight or surface area of the recipient.1-3 Flow cytometry is the method of choice to assess 
CD34+ cell content but protocols vary among institutions. Moreover, CD34 expression is merely a surrogate measure of hematopoietic stem cell or progenitor cell content. Nevertheless, enumeration of CD34+ cells is an essential tool of PBSC harvest and for comparing different mobilization or collection schemes. For successful enumeration of CD34+ cells, appropriate procedures are necessary to minimize pre-analytic, and post analytic variables. Within the realm of analysis, the hardware and software of a flow cytometry system are responsible for collecting data on individual cells and aggregating that data into useful information. Thus, a system should be chosen that is appropriate to the task, and its operator(s) should be well versed in the nuances of its hardware, software, and the inputs (cells, antibodies, gating protocols, etc) that the hardware and software manipulate Single-platform methodologies, in which a known number of wellcharacterized fluorescent beads are added to the analyte, are intended to be more reliable and easier to use than dual-platform methods, which combine data from a conventional hematology analyzer and a flow cytometer.11 Various platforms and methodologies have been compared,12-14 but prior results, regardless of outcome, should not discourage continued investigation and reporting of laboratory systems on which significant clinical outcomes depend. This test done on 2L8C flow cytometry systems, for the enumeration of CD34+ cells with both single platform and dual-platform methods.

\section{Aims}

To compare CD34+ cell counting results by using with and without the use of a separate hematology analyzer (ie, single-platform versus dual-platform methodologies).

\section{Material and Method}

A total of 34 samples of peripheral blood, apheresis packs, and cord blood were analysed and compared using the ISHAGE protocol with or without the addition of fluorescent microspheres.
Dual-platform CD34+ cell counting by $2 \mathrm{~L} 8 \mathrm{C}$ navious instrument.

\section{Comparison of single platform and dual- platform}

ISHAGE Methodology: Over a 11-month period, consecutive samples from patients at the M.Y.H. bone marrow unit at Indore M.P. Centre undergoing peripheral stem cell harvests after mobilisation for $5 \mathrm{~d}$ with G-CSF were analysed for CD341 cells by flow cytometry. A total of 34 samples of peripheral blood, apheresis packs, and bone marrow aspirate were studied. All samples were diluted to a leukocyte count of less than 1o million cell / sample dilution when necessary. Samples were analysed by the basic ISHAGE protocol (two-platform method) and the modified single platform ISHAGE method with the addition of a fluorescent bead (Stem-Kit, described below).

\section{Sample PreparationBasic ISHAGE method}

For each patient sample, four 12- $375-\mathrm{mm}$ tubes were labelled; 45/34 (nos. 1 and 2), WASH (no. 3 ), and 45/IgG (no. 4). Two millitres of Dulbecco's phosphate-buffered saline (DPBS;) were added to the WASH tube, and exactly $100 \mu \mathrm{l}$ of the well-mixed blood/apheresis sample were added to the bottom of the other tubes by using a repeater pipette. Leukocyte counts were determined on a Coulter STKS hematology analyser. If necessary, samples were diluted in DPBS to attain a leukocyte count (LKC) of 10 milion/ test. Ten microlitres of CD45 FITC and 10 $\mu 1 \mathrm{CD} 34 \mathrm{PE}$ were added to the first two tubes. Ten microlitres of CD45 FITC and $10 \mu \mathrm{L}$ PE IgG1 isotypecontrol were added to the fourth tube. All tubes were incubated at room temperature for 15 min in the dark and then $2 \mathrm{ml}$ ammonium chloride were added to lyse red blood cells. All tubes were gently vortexed and incubated at room temperature for $10 \mathrm{~min}$ in the dark, washed twice in DPBS (5 min at 500g), and resuspended in $1 \mathrm{ml}$ DPBS. Samples were then stored on ice at $4^{\circ} \mathrm{C}$ in the dark and analysed by flow cytometry within 1 h. 
Gating Strategy

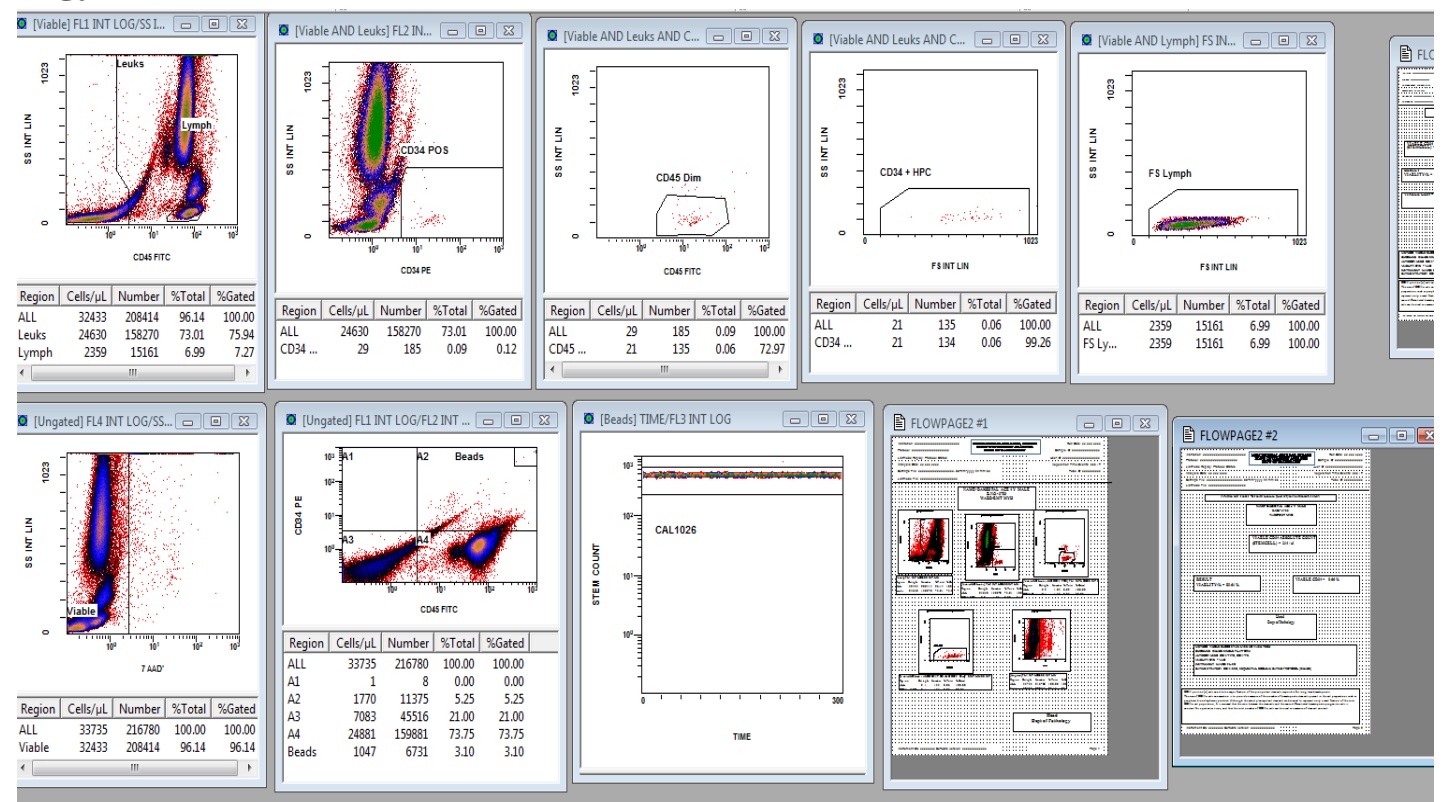

Basic ISHAGE protocol (Fig.). Flow cytometricanalysis was performed on a Coulter EPICStXL-MCL. Alignment and calibration was performed daily by using Coulter Flow-Checky and Flow-Setyfluorospheres. Compensation was checked visually with each run and adjusted by using Coulter CYTO-COMPycells when required.

Seventy-five thousand CD451 events were collected on histogram 2, with a minimum count of 100events in region (R) 4 on histogram 4. Region 5 on histogram 1 was set precisely to include only lymphocytes (bright CD45, low side scatter) and displayed on histogram 6 (FALS vs. side scatter). This helps to establish the minimum size range for the lymph-blast region (R4, histogram 6). Events such as platelets and other non specifically stained debris, if present, can be excluded. This region also helps to confirm that the FALS discriminator and FALS detector volts/gain are adequately set. The discriminator (or forward scatter threshold) was set to ensure that even the smallest CD451 lymphocytes scattered above it. FALS volts/gain were adjusted so that the smallest lymphocytes scattered around channel 200 of a 1,024 $3 \quad 1,024$ linear dot histogram. After determining the appropriate discriminator setting, R1 was positioned on histogram 1 to include all CD451 events. The lower extremity of R1 was set low enough to include all dim CD451 events (using histogram 5 as a guide). Histogram 5(CD34-PE vs. CD45FITC staining) helps to establish the lower limit of CD45 expression such that potential CD34cells (that express low levels of CD45) are not excluded. Histogram 2 was gated on R1. Region 2 was adjusted to intermediate side scatter. Histogram 3 was gated on R1 and R2. It is on this histogram that a cluster of cells appear, characterised by dim CD45 staining relative to lymphocytesand low side scatter. Region 3 was created as anamorphous region to best delineate the true CD341 cells from other events. Histogram 4 is gated on the cumulative regions $1-3$. The number of events in R4 is usually similar to that found in R3, but, if present, small platelet aggregates that stain weakly with both CD45 and CD34 (and are thus included in R3) can be excluded from R4 on the basis of light scatter. Both test (Fig. 1a) and control trol samples were analysed. A DPBS blank (tube 3) was run between the duplicate test (tubes 1 and 2) and the control tube (tube 4). The result from the two test samples was averaged, and the number of control IgG PE events was subtracted from the average CD341 events. For apheresis packs, this result was multiplied by the LKC and the pack volume in litres to give absolute CD341 cells 3 106/pack.

Basic ISHAGE protocol calculations. 5[average \#CD341 (R4)]62 Isotype control (R4) 
5(average CD451 Events Counted (R1)63 LKC (3109/L) 31000 CD34 10 milon /test

absolute number, the formula is as follows: Absolute Count (cells/ml)

5 Number of CD341 cells 3 Fluorospheres Concentration Number of Fluorospheres

The number of CD34 cells counted is R4. The number of fluorospheres counted is R6. The assayed Flow-Count Fluorosphere concentration is from the vial label. If the sample has been diluted before staining, the final answer must be multiplied by the dilution factor. To obtain the absolute CD341 cell number per apheresis pack, the absolute count per microlitre obtained is multiplied by the dilution factor, the volume of the pack (in litres), and by106 (to convert cells/ $\mu 1$ to cells 3 106/pack).

\section{Cell Viability}

In a separate experiment, the viability dye 7-AAD was added at a concentration of $1 \mu \mathrm{g} / \mathrm{ml}$ to a fresh and age dapheresis sample. A stock solution of $100 \mu \mathrm{g} / \mathrm{ml}$ in methanol was diluted $1: 100$ by adding $20 \mu \mathrm{L}$ of the 7-AADto the sample immediately after the addition of $2 \mathrm{ml}$ ammonium chloride lysing solution. Samples were analysed after $15 \mathrm{~min}$ of incubation, with 7-AAD being detected in the fourth PMT of the Epics XL flow cytometer. This allows not only a direct assessment of absolute number of CD341 cells but also discriminates between viable and nonviable CD341 cells.

\section{Results}

\section{CD34+ Counts, Single-Platform Method}

The number of CD34+ cells detected was slightly higher with the $2 \mathrm{~L} 8 \mathrm{C}$ navious than the for $\mathrm{WB}$ (median, 31.0/mL [range, 2.3-244.3/mL] versus median, 29.8/mL [3.4-234.9/mL]) and PBSCs (median,1040/mL [range, 67.5-14587.5/mL] versus median, 857.9/ $\mathrm{mL}$ [range, 50.6$13456.7 / \mathrm{mL}]$ ). These differences did not achieve statistical significance.

CD34+ Counts, Dual-Platform Method Differences in CD34+ cells detected with the navious $2 \mathrm{~L} 8 \mathrm{C}$ for $\mathrm{WB}$ (median, 31.0/mL [range,
2.2-220.5/mL] versus median, 30.4/mL [range 3.0-220.3/mL]) and PBSCs (median, 906.7/mL [range, 60.4-13433.4/mL] versus $912.3 / \mathrm{mL}$ [range, $44.2-12818.5 / \mathrm{mL}$ ]) were not statistically significant . Subtle differences in CD34+ cell counting between 2 systems and 2 methods did not achieve statistical significance. Assessment of Single-Platform and Dual-Platform Measurements No statistically significant differences were observed between the number of CD34+ cells when using either single-platform or dualplatform methods, regardless of the cytometer (Tables 4 and 5).CD34+ cell fraction (\%) correlated well by linear regression when comparing FACS Calibur and CytomicsFC500 data for both WB (r2 5 0.98) and PBSCs (r2 5 0.99), as shown in Figures 2 and 3, respectively. Similarly, CD34+ absolute counts correlated well when comparing single-platform and dualplatform analysis of WB with the NAVIOUS (r2 5 0.97, Figure 4), WB with PBSCs .

\section{Discussion}

It is now well established that engraftment success correlates with CD34+ cell count, independent of the degree of phenotypic similarity between donors and recipients. We owe this insight to the development of accurate cell counting methodologies, which are useful not only for individual patients but also for aggregating treatment and outcome data from many facilities. Flowcytometric quantification of CD34+ cells is now the most widely used tool to optimize mobilization and harvest of hematopoietic progenitor/stem cells for PBSC or bone marrow transplant.14 The first flow cytometric gold standard for the care of patients undergoing transplants was, in fact, a synthesis of data from 2 antecedent gold standards: the hematology analyzer for absolute leukocyte counting and the flow cytometer for the relative proportion of progenitor cells, as estimated by a surrogate marker such as CD34. Since the emergence of single-platform flow cytometric measurements, the original dual-platform standard has been 
criticized for being labor-intensive. Furthermore, historical data have linked the use of hematology analyzers to high coefficients of variation in CD4+ T-lymphocyteenumeration.16 Thus, the motivation for developing single-platform methods, using beads or volumetric techniques to generate absolute cell counts, is well

founded.17,18 Keeney et al11 adapted ISHAGE dual-platform guidelines for single-platform use, with favourable results. Several studies 19,20 have demonstrated that the single-platform approach achieves the highest possible reliability to quantify viable CD34+ cells, owing to reduced intra laboratory and inter laboratory variation. Therefore, this approach has been included in a variety of national 21 and international6 guidelines and a number of laboratory technical manuals. ${ }^{22,23}$ Nevertheless, CD34+ counting methodologies have not been exhaustively studied across the range of systems that are likely to be found in modern clinical pathology laboratories. To this end, we have navious, using both single-platform anddual-platform methodologies. Statistically significant differences would most likely cast single-platform methods in the most favorable light, whereas statistically insignificant differences would offer some reassurance that data from a wider variety of laboratories might be intelligently assimilated and analyzed. Differences between platforms and methodologies in our laboratory did not achieve statistical significance. This is in accordance with Sutherland et $\mathrm{al}^{14}$ who recently compared the $\mathrm{CD} 34+$ cell numbers derived from Stem-Kit and Tru Count systems and found that the 2 kits generated very similar data on arrange of fresh samples with BC navious.

\section{Conclusion}

It is the accurate determination CD34+ cell enumeration, properly validated, can support care for patients undergoing transplants setting. These modifications may improve the inter laboratory reproducibility. It can be argued that laboratories routinely using a single-platform method should be confident of alternative methods, not only for quality assurance but also for reliable patient care in the event that properly stored, in-date calibration beads are unavailable. Preliminary assessment of inter operator performance on replicate samples has been favorable in our laboratory but needs to be formalized as an ongoing assessment of intra laboratory variation for single plat form and dual-platform methods; we would welcome the opportunity to cooperate with other institutions to quantify inter laboratory variations. Altogether, these observations suggest that differences among flow cytometry systems need not deter laboratories from participating in local and multicenter transplant efforts. Multicenter investigations might corroborate this, or, alternatively, shed light on some as-yet unaccounted variables. As our laboratory only provides support foPBSC transplant, it would be instructive to know whether centers supporting bone marrow and/or cord blood transplant would reach similar conclusions as ours. "Negative" results in regard to differences among systems, methods, and laboratories would in fact be a positive outcome, indicating that consistent enumeration of CD34+ cells is achievable among laboratories.

\section{References}

1. Fritsch G, Stimpfl M, Kurz M, et al. Characterization of hematopoietic stem cells. Ann N Y Acad Sci. 1995;770:42-52.

2. Fritsch G, Stimpfl M, Kurz M, et al. The composition of $\mathrm{CD} 34$ subpopulations differs between bone marrow, blood and cord blood. Bone Marrow Transplant. 1996;17(2):169-178.

3. Allan DS, Keeney M, Howson-Jan K, et al. Number of viable CD34(+) cells reinfused predicts engraftment in autologous hematopoietic stem cell transplantation. Bone Marrow Transplant. 2002;29(12):967-972.

4. Chin-Yee I, Anderson L, Keeney M, Sutherland DR. Quality assurance of stem cell enumeration by flow cytometry; 
Canadian QASE Study Group. Cytometry. 1997;30(6):296-303.

5. Fritsch G, Printz D, Stimpfl M, et al. Quantification of CD34+ cells: comparison of methods. Transfusion. 1997;37(8):775-784.

6. Gratama JW, Orfao A, Barnett D, et al. Flow cytometric enumeration of CD34+ hematopoietic stem and progenitor cells; European Working Group on Clinical Cell Analysis. Cytometry. 1998;34(3):128-142.

7. Ikeda $\mathrm{K}$, Ohto $\mathrm{H}$, Kanno $\mathrm{T}$, et al. Automated programs for collection of mononuclear cells and progenitor cells by two separators for peripheral blood progenitor cell transplantation: comparison by a randomized crossover study. Transfusion. 2007;47(7):1234-1240.

8. Ikeda $\mathrm{K}$, Ohto $\mathrm{H}$, Nemoto $\mathrm{K}$, et al. Collection of MNCs and progenitor cells by two separators for PBPC transplantation: a randomized crossover trial. Transfusion. 2003;43(6):814-819.

9. Kessinger A, Sharp JG. Mobilization of blood stem cells. Stem Cells. 1998; 16(suppl 1):139-143.

10. Kessinger A, Sharp JG. The whys and hows of hematopoietic progenitor and stem cell mobilization. Bone Marrow Transplant. 2003; 31(5):319-329.

11. Keeney M, Chin-Yee I, Weir K, Popma J, Nayar R, Sutherland DR. Single platform flow cytometric absolute CD34+ cell counts based on the ISHAGE guidelines; International Society of Hematotherapy and Graft Engineering. Cytometry. 1998;34(2):61-70.

12. Barbosa IL, Sousa ME, Godinho MI, Sousa F, Carvalhais A. Single- versus dual-platform assays for human CD34+ cell enumeration. Cytometry. 1999;38(6): 274-279.

13. Gajkowska A, Oldak T, Jastrzewska M, et al. Flow cytometric enumeration of CD34+ hematopoietic stem and progenitor cells in leukapheresis product and bone marrow for clinical transplantation: a comparison of three methods. Folia HistochemCytobiol. 2006;44(1):53-60.

14. Sutherland DR, Nayyar R, Acton E, Giftakis A, Dean S, Mosiman VL. Comparison of two single-platform ISHAGE-based CD34 enumeration protocols on BD FACSCalibur and FACSCanto flow cytometers. Cytotherapy. 2009;11(5): 595-605.

15. Sutherland DR, Anderson L, Keeney M, Nayar R, Chin-Yee I. The ISHAGE guidelines for $\mathrm{CD} 34+$ cell determination by flow cytometry; International Society Of Hematotherapy and Graft Engineering. J Hematother. 1996;5(3):213-226.

16. Robinson G, Morgan L, Evans M, et al. Effect of type of haematology analyser on CD4 count. Lancet. 1992;340(8817):485.

17. Mercolino TJ, Connelly MC, Meyer EJ, et al. Immunologic differentiation of absolute lymphocyte count with an integrated flow cytometric system: a new concept for absolute $\mathrm{T}$ cell subset determinations. Cytometry. 1995;22(1):48-59.

18. Strauss K, Hannet I, Engels S, et al. Performance evaluation of the FACS Count System: a dedicated system for clinical cellular analysis. Cytometry. 1996;26(1):52-59.

19. Barnett D, Granger V, Kraan J, et al. Reduction of intra- and interlaboratory variation in CD34+ stem cell enumeration using stable test material, standard protocols and targeted training; DK34 Task Force of the European Working Group of Clinical Cell Analysis (EWGCCA). $\mathrm{Br} \quad \mathrm{J}$ Haematol. 2000;108(4):784-792.

20. Gratama JW, Kraan J, Keeney M, Sutherland DR, Granger V, Barnett D. Validation of the single-platform ISHAGE method for CD34(+) hematopoietic stem and progenitor cell enumeration in an 
international multicenter study.

Cytotherapy. 2003;5(1):55-65.

21. Barnett D, Janossy G, Lubenko A, Matutes E, Newland A, Reilly JT. Guideline for the flow cytometric enumeration of CD34+ haematopoietic stem cells: prepared by the CD34+ haematopoietic stem cell working party; General Haematology Task Force of the British Committee for Standards in Haematology. Clin Lab Haematol. 1999;21(5):301-308.

22. Gratama JW, Kraan J, Keeney M, Mandy F, Sutherland DR, Wood BL. Enumeration of Immunologically Defined Cell Populations by Flow Cytometry; Approved Guideline. Vol 27 (16). 2nd ed. Wayne, Pennsylvania: Clinical and Laboratory Standards Institute; 2007.

23. Sutherland DR, Keeney M, Gratama JW. Enumeration of CD34+ hematopoietic stem and progenitor cells. Curr Protoc Cytom. 2003; chapter 6:unit 6.4. 